\title{
Non facciamo le cose di prescia! (e nemmeno troppo di fretta!)
} Miriam Di Carlo

PUBBLICATO: 19 FEBBRAIO 2019

\begin{abstract}
Quesito:
Alcune persone ci chiedono informazioni riguardo la parola prescia, diffusa in alcune varietà regionali italiane. Qualcuno addirittura ci chiede se la parola derivi dall'inglese pressure 'pressione'.

Non facciamo le cose di prescia! (e nemmeno troppo di fretta!)

Diciamo subito che almeno in questo caso non siamo debitori all'inglese: la parola préscia 'fretta' è registrata come forma regionale centromeridionale nel GRADIT, come centromeridionale e arcaica nel Devoto-Oli 2018 e come centrale e meridionale nello Zingarelli 20rg. In realtà il tipo lessicale, nelle varianti pressa, pressa, pressia, presia, presa, è ben distribuito in tutta Italia e dunque anche nel Settentrione, fatta eccezione per alcune aree che illustreremo più avanti. La parola è riconducibile al verbo latino prèmere, il cui participio passato femminile prěssa $(m)$, divenuto poi in latino volgare "prěssia $(m)$, è passato a nome femminile assumendo una serie di significati: 'macchina per comprimere', 'calca di gente' e anche 'fretta'. Il nesso -ssj- di “prěssia $(m)$, ha dato prevalentemente -sc(i)-nelle varietà centrali e $-s(s i)$ - in quelle meridionali e settentrionali.
\end{abstract}

Sempre dal participio femminile prěssa $(m)$ deriva il verbo latino intensivo di prěmere, ovvero prěssare, da cui discende il sostantivo prěssione $(m)$ > pressione. Il fenomeno non è limitato all'italiano: la radice prěss-, infatti, rappresenta la base etimologica di una serie di parole diffuse nelle varietà romanze con un significato riconducibile a quello di 'premere':

I. it. pressione:

francese: pression; spagnolo: presión; catalano: pressió; portoghese: pressão; romeno: presiune;

2. it. pressa 'macchina che comprime', anche 'stampa':

fr. presse; sp. prensa; cat. premsa; port. prensa; rom. presa;

3. it. pressa 'calca di gente':

fr. presse; sp. ${ }^{\dagger}$ prisa; cat. ${ }^{\dagger}$ pressa.

Anche a prescia (e varianti) 'fretta' e 'ansia' nelle varietà italoromanze corrispondono il fr. presse con il significato 'ansia, angoscia', lo sp. prisa, il cat. pressa e il port. pressa 'mancanza di calma'.

Si nota come i significati di alcune delle forme citate, in italiano come nelle altre lingue considerate, siano estensioni metaforiche dell'idea di 'pressione' (originariamente espressa dal verbo latino): la pressa è una macchina che comprime, cosi come anche le macchine per la stampa (presse) erano dei rulli che esercitavano pressione, ispirati ai torchi che premevano l'uva per l'estrazione del succo. Nei luoghi molto affollati, la calca di gente genera una pressione forte, che spinge e che crea una vera e propria ressa (dal lat. rixa(m) 'litigio', incrociatosi con pressa). Infine la fretta non è altro che l'effetto di una pressione in termini di tempo che porta mancanza di calma e urgenza di agire (del resto già premere ha il significato estensivo di 'incalzare, urgere' e tra i vari significati di premura c'è anche quello di 'fretta').

Abbiamo fin qui confrontato le maggiori lingue romanze, ovvero quelle lingue che derivano dal latino. L'inglese, così come il tedesco, non è una lingua romanza ma nel corso dei secoli ha arricchito il lessico con parole derivanti dal latino. È il caso dell'inglese pressure 'pressione', press 'folla', 'stampa', 'fretta' (e tanti altri significati) che l'Oxford English Dictionary riconduce alla stessa base latina di cui si è parlato, su mediazione dell'italiano pressione e pressa: dunque è l'inglese che ha attinto dal latino su mediazione romanza, in questo caso italiana, e non 
viceversa. Se volessimo cercare un prestito inglese in italiano all'interno delle parole derivanti dalla base press-, troveremmo pressing, parola usata nel calcio ma anche in senso figurato 'pressione forte e insistente esercitata a livello psicologico'. Anche in questo caso, la base etimologica è il latino prěssa $(\mathrm{m})$, da cui, su mediazione dell'italiano pressa, deriva l'inglese to press, da cui a sua volta deriva il sostantivo pressing, arrivato in italiano con il significato calcistico.

Tornando a préscia, oggi la parola viene ricondotta per lo più alla varietà romanesca, a volte a quella siciliana e in generale alle varietà centro-meridionali. Ma guardando alla storia della parola nelle attestazioni antiche fino a quelle contemporanee ci accorgiamo che prescia (e varianti) era e continua a essere, con qualche rara eccezione, una parola in circolazione da nord a sud, su tutto il territorio italiano.

Le più antiche attestazioni di prescia 'fretta' risalgono ai testi delle origini e appartengono un po' a tutta la penisola: la prima è tratta da un testo anonimo probabilmente di area marchigiana, la seconda da un testo veneziano, la terza da un testo di mano dell'aretino Ser Gorello, la quarta da un testo abruzzese. Tranne la prima attestazione, che appartiene a un testo duecentesco, le altre fanno risalgono al Trecento:

Con grande pressça partese adlora lu Timore/ non çe feçe intervallu (La Giostra delle virtú e dei vizi, in Poeti del Duecento a cura di Gianfranco Contini, Milano, Riccardo Ricciardi, tomo II, r960, p. 9, fonte GDLI).

Et alguni sì coméli [è] asentadi, en gran prescia scomença a tajar pan et a meter vin en mujoli e par ke en una volta eli voja mançar e bever. (Fra Paolino Minorita, Trattato de regimine rectoris, a cura di Adolfo Mussafia, Vienna-Firenze, Tendler e Vieusseux, I868, p. 85, fonte TLIO).

Piacciate dir a me quel segui poscia, / e che fûr quesi chusciro a sì gran prescia,/ non curando erta né siepe né stroscia (Bartolomeo di Ser Gorello, Cronica dei fatti d'Arezzo, in Rerum Italicarum Scriptores a cura di Arturo Bini e Giovanni Grazzini, Bologna, Zanichelli, vol. XV, 1917-1933, fonte GDLI).

Quanno missere Phelippo se nne venne ad regire, / Calvacò tanto in presscia como chi in prescia à gire. /Lo conte nostro Lalle lo volse più sequire (Buccio di Ranallo di Popplito di Aquila, Cronaca aquilana rimata, a cura di Vincenzo De Bartholomaeis, Roma, Istituto Storico Italiano, 1907, p. 22I, fonte TLIO).

Nel Trecento troviamo il tipo lessicale, nelle varianti presa e pressa, anche in un testo del milanese Bonvesin da la Riva e, nella variante presia, in un testo di area veneta:

$\mathrm{Ni}$ trop impir la boca ni trop mangiar im presa; / Lo gord ke mangia im pressa, ke mangia a boca plena, / Quand el fiss appellao, el hav respond a pena. (Le opere volgari di Bonvesin da la Riva, a cura di Gianfranco Contini, Roma, Società Filologica Romana, 1941, p. 316).

quando elo ha dito queste parole, elo desmontà dala tore et sì va a gran presia inver lo palazo delo re ni ello non incontrà miga [...]et vigniva de sì gran presia como se la folgore et la tempesta lo chaçasse (Tristano Veneto, Il libro di messer Tristano, a cura di Aulo Donadello, Venezia, Marsilio, 1994, p. 305.327).

Il tipo lessicale derivante da *prěssia $(\mathrm{m})$ 'fretta' era dunque ben attestato nel Trecento nelle varietà italo-romanze: il tipo con-sc(i)- più diffuso in area centrale e meridionale mentre quello con-s(si)-, in area settentrionale. Anche nel Quattrocento prescia è presente in testi di area centrale, come ad esempio la Cronaca della città di Perugia dal I492 al I503 e nei testi del marchigiano Marco Scarsella da Tolentino (Macerata):

ovvero le dicevano con tanta prescia, che le mangiavano o lingiottivano, di maniera che non erano intesi da alcuno di astanti a quelli (Marco Scarsella da Tolentino, Giardino dei sommisti nel quale si dichiarano Dodecimila e più casi di coscienza, Venezia, Giacomo Antonio Samasco, 1595).

Prescia ricorre anche in area toscana: nel Trecento in Andrea da Barberino, nel Cinquecento nel poeta fiorentino Agnolo Firenzuola, in alcuni testi di area cortonese e nelle novelle del senese Pietro Fortini. Nel Seicento si trova anche nei testi dell'aretino Francesco Redi, del fiorentino Giovan Battista Fagiuoli e poi nell'Ottocento nel Guerrazzi. Nella traduzione delle epistole di Cicerone del senese Alessandro Maria Bandiera, risalente al Settecento, in particolare, prescia traduce le parole latine festinatio 'fretta, impazienza, precipitazione, velocità' e properatio 'fretta, premura' (Alessandro Maria Bandiera, L'epistole di Marco Tullio Cicerone a' familiari. In volgar toscano recate a riscontro del testo latino ed illustrate con note, Venezia, Tommaso Bettinetti, tomo I, I773). In area settentrionale si riscontra la parola nella commedia cinquecentesca Eutichia del mantovano Nicola Grasso, in 
testi di origine ferrarese, così come, nel Settecento, nelle lettere di San Paolo della Croce piemontese e in Goldoni che usa préscia in un testo italiano mentre nella variante préssa in una commedia in veneziano:

Rosalba: Con Filippino / Testè ci siamo coniugati in prescia (Carlo Goldoni, La scuola di ballo, Atto V, Scena X, in Tutte le opere di Carlo Goldoni [vol. VII de I Classici Mondadori] a cura di Giuseppe Ortolani, Milano, Mondadori, 1960, p. 342).

Barbara: Vorla andar via? Gh'ala tanta pressa? (Id., La buona madre: commedia veneziana in tre atti in prosa rappresentata la prima volta in Venezia nel Carnovale dell'anno 1761, Atto I, Scena VIII, in Tutte le opere di Carlo Goldoni [vol. VII de I Classici Mondadori] a cura di Giuseppe Ortolani, Milano, Mondadori, 1960, p. 662).

Ben diffusa su tutta la penisola nonostante le varianti sopra elencate, prescia è inserita nel Vocabolario degli Accademici della Crusca nella III (I69I) e nella IV edizione (I729-I738) con un solo esempio del Firenzuola. Il Tommaseo-Bellini, pur registrandola assieme al verbo presciare 'far prescia, sollecitare' che dichiara ormai in disuso, precisa che "vive in qualche dial[etto]".

Oggi la situazione vede la vitalità di prescia nel Centro Italia, eccezion fatta come vedremo, per la Toscana: è registrata in dizionari dialettali marchigiani, in quelli dei centri umbri di Spoleto, Foligno e Orvieto e della Tuscia laziale e in tutti i repertori del romanesco. Per quanto riguarda il romanesco, inoltre, prescia ha una storia letteraria che risale a Berneri a Peresio fino a Belli e Trilussa. La vitalità di prescia nel romanesco contemporaneo è ben testimoniata dalla poesia di Aldo Fabrizi Amatriciana mia e dai versi di Mauro Milesi:

E ammalapena er sugo fa locchietti,/ assieme a pecorino e parmigiano,/ conditece de prescia li spaghetti (cit. tratta da Loredana Tartaglia, Regina Amatriciana, Repubblica.it, 21/6/20II).

Dice l'Unno che vedé San Pietro/in prescia in prescia fa du' passi addiètro/ e er zu cavallo pare che se stenne (cit. tratta da Stefano Clerici, La vita dei papi? Un vero poema, Repubblica.it, 19/1/2004).

A proposito della varietà regionale romanesca, prescia ricorre anche nelle Storie di Ascanio Celestini:

e appena mi capacito torno di prescia al carretto e attacco a resuscitare tutti l'altri morti [...]. Mi azzitto e mi metto a camminare per la campagna. Cammino e perdo la strada ma non cho prescia di ritrovarla (Ascanio Celestini, Storie di uno scemo di guerra: Roma, 4 giugno 1944, Torino, Einaudi, 2005, pp. 56-58).

Nel Settentrione, oggi prevalgono le varianti préssa-presa-presia che sono registrate in dizionari di area friulana, veneziana, padovana, triestina, vicentina, ma anche bergamasca, milanese, lecchese, genovese. La forma prescia viene usata prevalentemente in area piemontese, attestata sia nei repertori lessicografici, sia in giornali come il "Biellese" e l"Eco del Risveglio", in contesti dialettali o di italiano regionale:

Fa poi pessima impressione nella cittadinanza la prescia dell'Eco che, annunziando linchiesta, afferma già subito che $i$ primi risultati sono favorevoli al personale ([s.f.], Sulle cose del manicomio di Novara, in "Il Biellese" consultabile in giornalidelpiemonte.it, $\mathrm{XVII}(20)$, 1903, p. I).

"Bruno, fermat un atim...!" "No a gò prescia...A gò da nà..." (No ho premura...devo andare) (Giancarlo Castellanoßruno, "'é propi l'ora da nà", in "Eco del Risveglio" consultabile in giornalidelpiemonte.it, 27/7/2017, p. II).

Muovendo verso sud si hanno riscontri anche nel dialetto di Modena, di Bologna e, in generale, nelle varietà reggiane.

Nel Meridione è diffusa prevalentemente, ma non esclusivamente, la forma prescia. Ad esempio a Napoli si ha prescia e press $\boldsymbol{\partial}$ da cui anche mpress $\boldsymbol{\partial}$ mpress $\boldsymbol{\partial}$ 'veloce veloce'. La fiesolana Dacia Maraini, avvertendo prescia come parola napoletana, la usa nella commedia Donna Lionora giacobina, sia per caratterizzare la parlata di alcuni personaggi napoletani popolani (come Gennaro e Peppina) e no (come il cardinale Rufo):

GENNARO: Il generale non può aspettare, tiene prescia. PEPPINA: Io tengo chiù prescia d'isso. G: A sua prescia è chiù importante da a tua. P: E pecché? G: La sua è na prescia generale, na prescia francese, na prescia rivoluzzionaria! P: E la mia che prescia è? G: Na prescia morta e famine...te saluto Peppina, addio! [...] RUFO: Nola è nostra... domani tocca a Napoli... scrivete mprescia mprescia Gaetano (Dacia Maraini, Maria Staurda. Mela, Donna Lionora giacubina, Stravaganza, Un treno, Una notte, Milano, Rizzoli, 200I, p. 2I. 28). 
La parola è diffusa in area abruzzese e molisana, così come in Basilicata e in Calabria, in cui si registrano anche mpresciusu, prescialoru, 'frettoloso' e i verbi presciäri, mpresciàri, spresciàra, spresciari 'affrettare, sollecitare'. Pochi riscontri, eccezion fatta per alcune zone del Salento, in area pugliese dove si preferiscono altri tipi lessicali. In Sardegna (almeno nei repertori consultati, gallurese e logudorese) si ha la parola presse 'fretta' da cui pressighinósu 'frettoloso', che viene variamente impiegata in una serie di proverbi:

Chi cha presse faghed aju chi ha troppa fretta perde più tempo. Robba fatta in presse, bessid a piliesse cosa fatta in fretta riesce male, al rovesciol in presse, de presse in fretta. Bae in presse, de presse a sa é su duttore e a sa é su preideru va in fretta dal medico e dal prete (Pietro Casu e Giulio Paulis, Vocabolario Sardo-Logudorese-Italiano, Nuoro, ILISSO, 2002).

Allo stesso modo, prescia è ben attestata in Sicilia: i dizionari dialettali siciliani riportano sistematicamente il lemma con tutti i suoi derivati. A livello letterario prescia viene usata nella varietà regionale siciliana delle novelle Perdutamente di Luigi Capuana ma anche nelle battute in siciliano della commedia Liolà di Pirandello:

DON SIMUNI: Esempri cu sta mmallita prescia vu'! ZA' CRUCI Prescia? Chi prescia? Si la stavano manciannu tutta! Idda ca su quattru coccia! E sperti, taliatilu, sugnu i' chaju 'a prescia! [...] DON SIMUNI Mi dissi: - "Mallita la prescia!" (Luigi Pirandello, Liolà, commedia campestre in 3 atti, Roma, Formaggini, 1917, p. 122.124).

Anche Sciascia nel Giorno della civetta usa la parola, che compare, per citare un esempio contemporaneo, in moltissimi romanzi di Andrea Camilleri la cui lingua, che ha come base l'italiano, viene infarcita di tratti tipicamente siciliani, spesso attinti dal lessico.

Nell'archivio della "Repubblica" si riscontrano una serie di articoli che impiegano la parola facendo riferimento alla Sicilia:

arriminati! Che corrisponde a "datti una mossa, sbrigatil". Non sanno che la prescia è per il siculo, pessima consigliera e sgradita ospite (Pippo Russo, La missione impossibile di spiegare l'arriminare, Repubblica.it, 2/12/2009.

"Dovevo uscire dal mio territorio" continua l'artigiano che lavora con cinque persone ed ha aperto un nuovo laboratorio nel centro di Modica. "A prescia amapara a curriri, si dice dalle nostre parti. Ecco la fretta mi ha fatto correre il cervello" (Giorgio Ruta, Disegno i piatti per Cracco, lui mette "soltanto" il cibo, Repubblica.it, I7/4/2018; si noti la presenza dell'equivalente italiano fretta).

Come conferma, oggi in una località vicino Palermo si possono assaggiare le sfince ri prescia ovvero 'le sfince fatte di fretta', che prevedono una ricetta semplificata, mentre dal repertorio dei proverbi siciliani di Pitrè scopriamo che per un siciliano:

Cu' havi prescia, è megghiu ca si cura ('chi ha fretta, è meglio che si curi') (Giuseppe Pitrè, Proverbi siciliani, vol. III. Palermo, Pedone Lauriel).

Rimane fuori dall'area di diffusione diprescia (e forme affini) la Toscana: nessuno dei dizionari dialettali di Toscana, tranne quello maremmano, che però coinvolge anche centri laziali, riporta il lemma. Dalla carta AIS I606 si vede chiaramente che il tipo prescia si arresta al confine toscano lasciando posto ai tipi furia e fretta. Tale dato è confermato anche dai dati dell'inchiesta LinCi ( La Lingua delle Città), che riporta furia e fretta per le città di Arezzo, Carrara, Firenze, Livorno, Lucca, Massa, Pisa, Pistoia, Prato, Siena. Solo a Grosseto, la cui varietà si avvicina di più a quelle centrali, si ha una sola attestazione con l'iniziale sonorizzata: bréscia.

Concludendo, la prěssia $(\mathrm{m})$ latina, che sopravvive oggi nelle maggiori lingue romanze europee ed è entrata anche in inglese, a torto viene considerata base di derivazione della sola prescia in romanesco e nelle varietà centrali: infatti da prěssia $(m)$ derivano molte varianti che, sempre con il significato di 'fretta', sono ben diffuse in molte altre varietà italo-romanze, anche settentrionali e meridionali, eccezion fatta per la Toscana e poche altre zone. Nello scritto e in contesti formali senz'altro preferibile ricorrere allo standard fretta.

\section{Cita come:}

Miriam Di Carlo, Non facciamo le cose diprescia! (e nemmeno troppo difretta!) , "Italiano digitale", 2019, VIII, 2019/1 (gennaio-marzo) , pp. 23-27. 
DOI: 10.35948/2532-9006/2019.3069

Copyright 2019 Accademia della Crusca

Pubblicato con licenza creative commons CC BY-NC-ND 